\title{
Synthesis and Biological Assay of GSH Functionalized Fluorescent Quantum Dots for Staining Hydra vulgaris
}

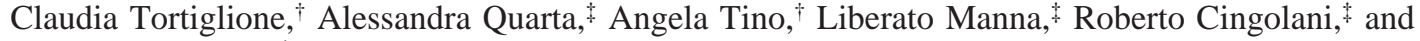 \\ Teresa Pellegrino*, \\ CNR, Istituto di Cibernetica "E Caianiello", Via Campi Flegrei, 34, 80078 Pozzuoli, Italy, and National \\ Nanotechnology Laboratory of CNR-INFM, Unità di ricerca IIT and Scuola Superiore ISUFI, Via per Arnesano, \\ 73100 Lecce, Italy Received November 12, 2006; Revised Manuscript Received January 31, 2007
}

\begin{abstract}
Quantum dots (QDs) have been used extensively as fluorescent markers in several studies on living cells. Here, we report the synthesis of conjugates based on glutathione (GSH) and QDs (GSH-QDs) and we prove how these functionalized fluorescent probes can be used for staining a freshwater invertebrate called Hydra vulgaris. GSH is known to promote Hydra feeding response by inducing mouth opening. We demonstrate that GSH-QDs as well are able to elicit biological activity in such an animal, which results in the fluorescent staining of $H y d r a$. GSH-QDs, once they reach the gastric region, are internalized by endodermal cells. The efficiency of GSH-QD internalization increases significantly when nanoparticles are coadministrated with free GSH. We also compared the behavior of bare QDs to that of GSH-QDs both in the presence and in the absence of free GSH. The conclusions from these series of experiments point to the presence of GSH binding proteins in the endodermal cell layer and uncover a novel role played by glutathione in this organism.
\end{abstract}

\section{INTRODUCTION}

Colloidal semiconductor nanocrystals, also known as quantum dots (QDs), are emerging as a new class of inorganic fluorophores for imaging living and fixed cells in fluorescence microscopy (1). The QD probes have unique photophysical properties, such as size-tunable emission spectrum, narrow emission peak, broad absorption profile, and high brightness. In addition, QDs are much more stable to the permanent loss of fluorescence than conventional organic fluorophores $(2,3)$. Therefore, they offer several advantages over standard organic dyes for multicolor, long-term, and high-sensitivity fluorescence imaging.

Recently, several strategies have been developed to deliver stable, water-soluble quantum dots (4). These procedures allow also to introduce on the outmost shell of the QDs specific anchoring points by which biomolecules can be conjugated to the nanoparticles. In the latest years, it has been demonstrated that these new bioconjugates are useful for applications in many of the classical immunocytochemistry protocols, as well as for in vivo staining of living cells on higher vertebrates (5). On the other hand, no studies of cell tracking, migration, or physiological response using QD functionalized with bioactive molecules have been reported on less-evolved organisms.

The tripeptide glutathione ( $\gamma$-L-glutamyl-L-cysteinylglycine, GSH) has been well-known to biochemists for generations. Both the reduced form (GSH) and its oxidized dimer (GSSG) have been implicated in a variety of molecular reactions throughout the animal kingdom. Although it is best known for its role as a free radical scavenger, GSH also performs a number of other functions in cell survival and metabolism, including amino acid transport, detoxification of xenobiotics, maintenance of protein redox state, neuromodulation, and neurotransmission (6). Almost

* To whom correspondence should be addressed. teresa.pellegrino@ unile.it.

† Istituto di Cibernetica "E Caianiello".

\#ational Nanotechnology Laboratory of CNR-INFM, Unità di ricerca IIT and Scuola Superiore ISUFI.
50 years ago, Loomis and Lenhoff (7) suggested a role of GSH in signal transduction in the coelenterate Hydra. Hydra, together with coral, sea anemones, and jellyfish, are members of the primitive phylum Cnidaria. It is shaped as a "two-ply hollow tube", made up of both outer (ectodermal) and inner (endodermal) epithelial layers. The mesoglea, an extracellular matrix, separates the two epithelia. At the posterior end of the tube, there is a basal disk with which the Hydra usually attaches to a surface, whereas at the anterior end, there is a mouth surrounded by a ring of tentacles (Figure SI1, Supporting Information). The tentacles are armed with many nematocytes, which contain nematocysts (stinging capsules). The latter start the feeding process by reverting a coiled tube, which pierces and wounds the prey. In the absence of prey, GSH is the physiological stimulator of the feeding behavior (8), by inducing tentacle contraction and mouth opening. Grosvenor et al. (9) and Bellis et al. (10) described a class of binding sites for GSH in Hydra that provides the basis for the behavioral response.

Hydra is a model organism that offers several potential advantages as an investigation target by means of QDs, such as (i) the simplicity of the body structure, which allows easy detection of fluorescent probes; (ii) the dynamics of the two epithelia, as Hydra cells, which continuously divide, migrate and are eliminated at the apical and basal ends of the polyp, allow cell migration and tracking studies; and (iii) the regenerating capability, involving extensive remodeling and plasticity phenomena, whose temporal dynamics can be best approached by using photostable fluorescent probes. Finally, and most importantly, Hydra allows us to validate by a reliable assay the biological activity of GSH-conjugated QDs.

In this report, we describe the synthesis and the characterization of core-shell $\mathrm{CdSe} / \mathrm{ZnS}$ QDs conjugated to glutathione for the staining of Hydra. The biological activity evoked by GSH-QDs in living Hydra animals was also studied. We found that cells lining the gastric cavity were able to internalize the bare QDs in the presence of reduced GSH in the media bath, as intense fluorescence was present in this cell type. Interestingly, in the same conditions, the efficacy of the internalization 
doubled in the presence of GSH-coated QDs. These results demonstrate the involvement and the activation of GSH-specific endodermal cell uptake mechanisms in Hydra, such as those mediated by carriers, specific receptors, or membrane metabolic enzymes into any type of living cells. GSH-functionalized nanoparticles represent the first example of fluorescent and bioactive GSH adduct suitable for specific staining of one type of tissue on Hydra.

\section{MATERIALS AND METHODS}

Chemicals. (1-Ethyl-3-(3-dimethylaminopropyl)carbodiimide hydrochloride) (EDC), $\gamma$-L-glutamyl-L-cysteinylglycine (GSH), 5,5'-dithiobis(2-nitrobenzoic acid) (Ellman's reagent), and 4',6diamidino-2-phenylindole dihydrochloride (DAPI) were purchased from Aldrich. $O, O^{\prime}$-Bis(2-aminoethyl)octadecaethylene glycol, diamino-PEG-897) was purchased from Fluka. DLDithiothreitol (DTT) was purchased from Biochemika. $\mathrm{N}$ Hydroxysuccinimide (NHS) was purchased from Pierce. Agarose gel EEO was purchased from Eppendorf. All solvents that we used had purity of analytical grade.

Synthesis of Water-Soluble Quantum Dots. TOP/TOPOcapped $\mathrm{CdSe} / \mathrm{ZnS}$ core/shell QDs were prepared by following standard colloidal synthesis procedures $(11,12)$. For fluorescence imaging, given the Hydra autofluorescence, we found it more convenient to work with QD samples with an emission band peaked at wavelengths longer than $590 \mathrm{~nm}$. As-synthesized QDs are hydrophobic, and therefore, they were transferred into water by means of a standard procedure based on wrapping them within an amphiphilic polymer shell, which had been developed in a previous work by our group (13). The resulting polymercoated particles (PC-QDs) were purified twice by highperformance liquid chromatography HPLC (Agilent1100 on a Sephacryl S-300 HR column, using $150 \mathrm{mM} \mathrm{NaCl}$ in $50 \mathrm{mM}$ phosphate buffer at $\mathrm{pH} 7.0$ as mobile phase). These purified PC-QDs have carboxyl groups dangling at the outer surface of the polymer layer. The concentration of the quantum dots was determined by measuring their optical absorption spectra, as their molar extinction coefficient is know from the literature (14). As an example, the extinction coefficient of a quantum dot sample with the first exciton peak centered at $590 \mathrm{~nm}$ was estimated as $258050 \mathrm{~cm}^{-1} \mathrm{M}^{-1}$ by extrapolation from the published curve (14).

Functionalization of Quantum Dots with Diamino-PEG $897\left(\mathbf{N H}_{2}-\mathbf{P E G}-\mathbf{N H}_{2}\right)$. The buffer of the HPLC-purified, PCQDs was exchanged with $50 \mathrm{mM}$ sodium borate buffer $(\mathrm{pH}$ 9.0) by using a centrifuge tube (MWCO 30000 ). The same buffer was used to dissolve diamino-PEG $\left(\mathrm{NH}_{2}-\mathrm{PEG}-\mathrm{NH}_{2}\right)$ with molecular weight equal to 897.10. For the coupling experiments, one of the two amine groups of the diamino-PEG molecules was linked to one of the carboxyl groups present on the PC-QDs. This was achieved by using EDC as cross-linker, which induced amide bond formation (15). Interparticle crosslinking, due to the bridging of one or more diamino-PEG molecules between two PC-QDs via both terminal amine groups, was negligible at ratios of diamino-PEG molecules to PC-QDs equal to 500:1 or lower. Therefore, a 500:1 ratio was used in all experiments.

In a typical procedure, a buffer solution of $6 \mu \mathrm{M}$ PC-QDs and a solution of $3 \mathrm{mM}$ diamino-PEG were mixed together, after which a solution of $600 \mathrm{mM}$ EDC was added to reach an excess molar ratio of EDC to PC-QDs equal to $10^{5}: 1$. The resulting solution was incubated for $3 \mathrm{~h}$ under vigorous stirring. To remove the excess of unreacted diamino-PEG, the solution was filtered several times through centrifuge tubes (MWCO 30 000). Diamino-PEG-functionalized QDs (PEG-QDs) were resuspended in water at a $6 \mu \mathrm{M}$ final concentration.

Conjugation of GSH to Quantum Dots. GSH has two free carboxyl groups and a free thiol group. The $\mathrm{COOH}$ groups can be exploited to link the GSH to the PEG-QDs via formation of an amide bond with the free amino groups of the diaminoPEG molecules bound to the polymer shell that encases the PCQDs. In detail, to a $6 \mu \mathrm{M}$ solution of PEG-QDs dissolved in sodium borate buffer ( $\mathrm{pH} 9.0)$, a $10 \mathrm{mM}$ GSH solution dissolved in the same buffer was added, to reach a molar excess of GSH per PEG-QD equal to 1700:1. Borate buffer solutions containing EDC $100 \mathrm{mM}$ and NHS $75 \mathrm{mM}$, respectively, were then added. The resulting solution was incubated at room temperature for $3 \mathrm{~h}$ under vigorous stirring. To remove the unreacted GSH, the sample was washed five times on $20 \mathrm{~mL}$ centrifuge filters (30 $000 \mathrm{MWCO}$ ). In general, for the experiments carried out on Hydra, the sample was then reconcentrated on the filter to reach a final concentration equal to $6 \mu \mathrm{M}$.

Characterization by Gel Electrophoresis. Prior to gel electrophoresis, to each sample a solution containing Orange $\mathrm{G}$ and $30 \%$ glycerol in gel-loading buffer corresponding to $20 \%$ of the sample volume was added. $2 \%$ agarose gels were prepared in $0.5 \times$ TBE buffer. The electrophoretic run was carried out for $60 \mathrm{~min}$ at $100 \mathrm{~V}$. After the run, the gel was observed under UV light.

Ellman's Test and DTT Treatment. To assess the amount of GSH bound to the PEG-QDs, Ellman's assays were carried out (16). This procedure allowed us to quantify the $\mathrm{SH}$ groups of the glutathione. The Ellman's reagent, namely, 5,5'-dithiobis(2-nitrobenzoic acid), reacts with free thiol groups $(\mathrm{SH})$ and releases a chromogenic compound (5-thio-2-nitrobenzoic acid) that can be detected easily by means of its optical absorption band peaked at $412 \mathrm{~nm}$. The absorbance at this wavelength is therefore directly proportional to the concentration of $-\mathrm{SH}$ groups in solution. Due to the broad optical absorption spectrum of the QDs, the absorption region of the 5-thio-2-nitrobenzoic acid is covered and the absorbance of this chromogen could not be read directly in the presence of GSH-QD. In order to overcome this inconvenience, after the Ellman's reaction the sample solution was centrifuged in cutoff filters in order to separate the yellow chromogen (which passed easily through the membrane) from the GSH-QD conjugates (which did not pass through the pore membrane).

The concentration of thiol groups in solutions of GSH-QDs was determined by extrapolation from a calibration curve that was built by carrying out the Ellman's test on free GSH solutions at known concentrations. Ellman's tests were actually carried out both on solutions of GSH-QDs conjugates and on the same solutions after they had been treated with a $10 \mathrm{mM}$ solution of dithiothreitol (DTT). DTT is able to cleave the disulfide bonds to form free $-\mathrm{SH}$ groups (17). The DTT treatment was applied routinely to both $\mathrm{PEG}-\mathrm{QDs}$ and $\mathrm{GSH}-\mathrm{QDs}$ samples immediately prior to the in vivo experiments. In a typical procedure, a solution of $10 \mathrm{mM}$ DTT was added to a $6 \mu \mathrm{M}$ solution of GSH-QDs. The resulting mixture was left under stirring for $3 \mathrm{~h}$, after which the sample was centrifuged in 30000 MWCO centrifuge filters in order to remove the excess of free DTT. At least five washes were carried out before redissolving the sample in Hydra physiological solution (Hy Sol: $\mathrm{CaCl}_{2}, 1$ $\mathrm{mM}$; and $\mathrm{NaHCO}_{3}, 0.1 \mathrm{mM}$ ).

Growth and Maintenance of Hydra. Hydra vulgaris (strain Zurich, originally obtained by P. Tardent) were asexually cultured in physiological solution by the method of Loomis and Lenhoff (7) with minor modifications. The animals were kept at $18 \pm 1{ }^{\circ} \mathrm{C}$ and fed three times per week with freshly hatched Artemia salina nauplii. All animals were starved $24 \mathrm{~h}$ prior to the experiments.

In Vivo Experiments. Groups of 20 animals were collected in plastic multiwells, allowed to equilibrate at room temperature in physiological solution (Hy Sol) buffered with $1 \mathrm{mM}$ Tris$\mathrm{HCl}$ (pH 7.4). The test was initiated by adding $300 \mu \mathrm{L}$ of $\mathrm{Hy}$ 
Sol containing either PEG-QDs or GSH-QDs at different concentrations (ranging from 3 to 300 nanomolar) to each well containing 20 polyps. One hour after incubation, the final volume was brought to $1 \mathrm{~mL}$ and the animals were kept at $18{ }^{\circ} \mathrm{C}$ for $24 \mathrm{~h}$. For each QD concentration tested, a parallel trial was performed by adding GSH $10 \mu \mathrm{M}$. $24 \mathrm{~h}$ after incubation, the samples were rinsed in Hy solution several times before observation by fluorescence microscopy.

Fluorescence Microscopy. In vivo experiments were monitored for the first hour under a fluorescence stereomicroscope (Olympus ZSX-RFL2) equipped with a GFP filter set (BP460490/DM505/LP510). In vivo fluorescence imaging of the whole animals and dissociated cells, along with imaging of fixed tissue sections, was accomplished by using an inverted microscope (Axiovert 100, Zeiss) equipped with a digital color camera (Olympus, DP70) and fluorescence filter sets (BP450-490/ FT510/LP515). For imaging acquisition and analysis, the software system Cell F (Olympus) was used.

Hydra Tissue Dissociation and Cryosectioning. Hydra polyps were dissociated according to Flick and Bode (18) with minor modifications. This procedure allows dissociation of Hydra tissue into a suspension of viable cells. Briefly, test animals were rinsed with $\mathrm{Hy} \mathrm{Sol}$ and soaked in the dissociation solution for $60 \mathrm{~min}$ at $0{ }^{\circ} \mathrm{C}$. Following a mechanic dissociation by a narrow glass pipet, the suspensions containing dissociated cells were transferred on microscope slides for fluorescence microscopy.

For tissue sectioning, test animals were fixed in $4 \%$ paraformaldeyde, $\mathrm{pH}=7.4$, at $4{ }^{\circ} \mathrm{C}$, rinsed three times in phosphate saline buffer (PBS: $8 \mathrm{~g} / \mathrm{L} \mathrm{NaCl} ; 0.2 \mathrm{~g} / \mathrm{L} \mathrm{KCl} ; 1.44 \mathrm{~g} / \mathrm{L} \mathrm{Na}_{2}-$ $\mathrm{HPO}_{4} \bullet 7 \mathrm{H}_{2} \mathrm{O} ; 0.24 \mathrm{~g} / \mathrm{L} \mathrm{KH}_{2} \mathrm{PO}_{4}$ ), soaked overnight in $30 \%$ saccharose in PBS and then embedded in the frozen section medium Neg-50 (Richard-Allan Scientific). Cryosections of 10 $\mu \mathrm{m}$ thickness were obtained by a cryostat (Leitz, digital 1760), collected on gelatin-coated slides (Superfrost microscope slides, Menzel), and mounted with Aquatex mounting medium (Merk) before imaging. For cell nuclei detection, sections were stained with 4',6-diamidino-2-phenylindole dihydrochloride (DAPI) 1 $\mathrm{mg} / \mathrm{L}$ before mounting.

\section{RESULTS}

Synthesis of Glutathione-Quantum Dots Conjugates. In Figure 1A, a sketch of the GSH-QDs conjugates is shown. We transfer hydrophobic TOP/TOPO capped $\mathrm{CdSe}-\mathrm{ZnS}$ nanocrystals into water by wrapping the QDs in an amphiphilic polymer (poly(maleic anhydride-alt-1-tetradecene)) and then by cross-linking this polymer shell using bis(6-aminohexyl)amine (13). The resulting water-soluble polymer-coated quantum dots have a negatively charged surface, due to the hydrolyzed carboxyl groups present at the surface of the polymer shell. Therefore, these nanoparticles migrate from the negative pole toward the positive pole in the gel electrophoresis run (Figure $1 \mathrm{~B}$, lane 1). The carboxyl groups are then used to link diaminoPEG molecules through amide bond formations via the crosslinker EDC. The diamino-PEG molecules are introduced because they stabilize more effectively the nanoparticles in the Hydra solution and also because they introduce amino groups at the surface of the QDs. These amino groups in turn are used to link the tripeptide glutathione (GSH) through an additional amide bond formation. In a previous study, it was demonstrated by some of us that it is possible to tune the number of PEG molecules attached per nanoparticle by varying the length of the PEG molecule and the ratio of EDC added per molecule (19). In the present case, to achieve a dense surface of glutathione molecules attached per QDs, we have chosen PEG molecules of molecular weight equal to 897. At this length, it is possible to pack the surface of the quantum dots with a

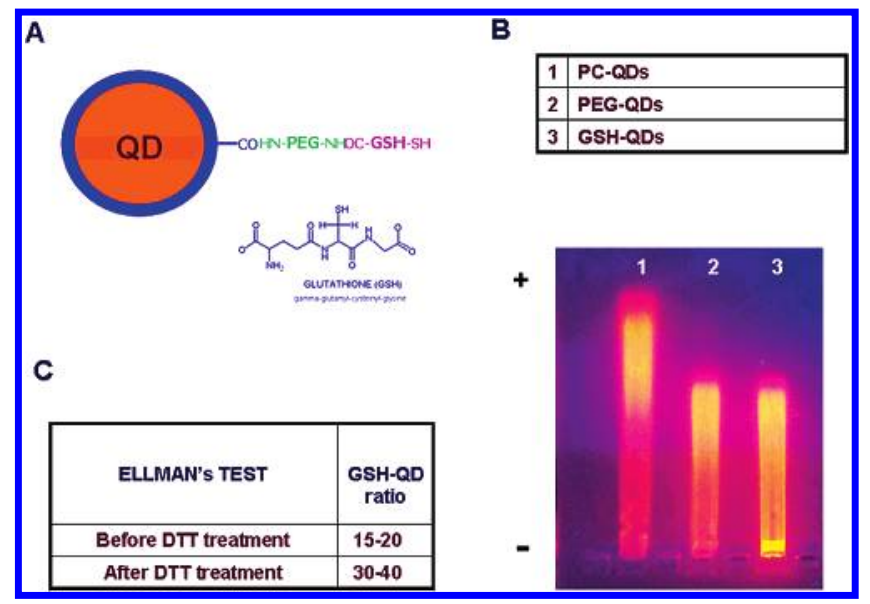

Figure 1. (A) Schematic representation of a GSH-QD conjugate. Polymer-coated quantum dots were first conjugated to diamino-modified PEG molecules and then to GSH through amide bond formation. (B) Characterization of the GSH-QD conjugates by means of gel electrophoresis. The samples loaded on the bottom part of the gel move through the gel from the negative to the positive pole. Lane 1 represents the migration of the polymer-coated quantum dot (PC-QD). Lane 2 corresponds to the migration of polymer-coated quantum dot functionalized with diamino PEG molecules having a molecular weight of 897 (PEG-QD). Lane 3 corresponds to the migration of PEG-coated quantum dots bound to GSH molecules (GSH-QD). (C) Evaluation of the number of GSH attached per nanoparticle. Ellman's test allows one to detect the number of thiol groups and therefore the cysteine groups attached per quantum dot. The measure was repeated on GSHQD samples before and after DTT addition.

saturated number of PEG molecules (19). This prevents the nanoparticles from precipitating (as they become highly stable), and in addition, it ensures a saturated number of amino groups attached per PC-QDs. To verify the linkage of the PEG molecules to the QDs, we used gel electrophoresis. The PEGQDs still move toward to the positive pole, although they are visibly retarded with respect to the original polymer-coated QDs (Figure 1B, lane 2). Once the glutathione is attached to the PEG-QDs, these dots have almost the same mobility on the gel as the starting PEG-QDs, although the front edge of the band is slightly retarded with respect to the latter (Figure 1B, lane 3), a behavior that can be attributed to the attachment of the small gluthatione tripeptide to the PEG-QDs.

Furthermore, the GSH-QD band also shows a partial precipitation in the loading well. This behavior is very likely due to the formation of interparticle $\mathrm{S}-\mathrm{S}$ bridges that could form among some GSH-QDs. This hypothesis is supported by the Ellman's test performed on GSH-QD samples before and after treatment with a reducing agent such as DTT. Statistically, the number of free thiol groups and therefore of reduced GSH molecules attached per QD doubles after treatment with DTT (Figure 1C).

We have also characterized the various steps of the conjugation reaction by FT-IR measurements. By checking the absorption spectra of the polymer-coated QDs, the PEG-QDs, and the GSH-QDs, respectively, a significant change of the corresponding profiles was observed. In the spectra corresponding to the GSH-QDs, a new peak appeared at $1776 \mathrm{~cm}^{-1}$. While the assignment of this new peak is not obvious, we believe that it can be ascribed to the formation of a new amide bond between the carboxy group of the GSH and the amino group of a PEG molecule present on the surface of the PC-QDs (see Supporting Information, Figure SI2).

One critical aspect of the linking reaction is that it must ensure that the thiol groups of the cysteine are still present in the reduced form after the attachment to the dots. Lenhoff and Bovaird (20) showed in fact that Hydra recognizes the specific 


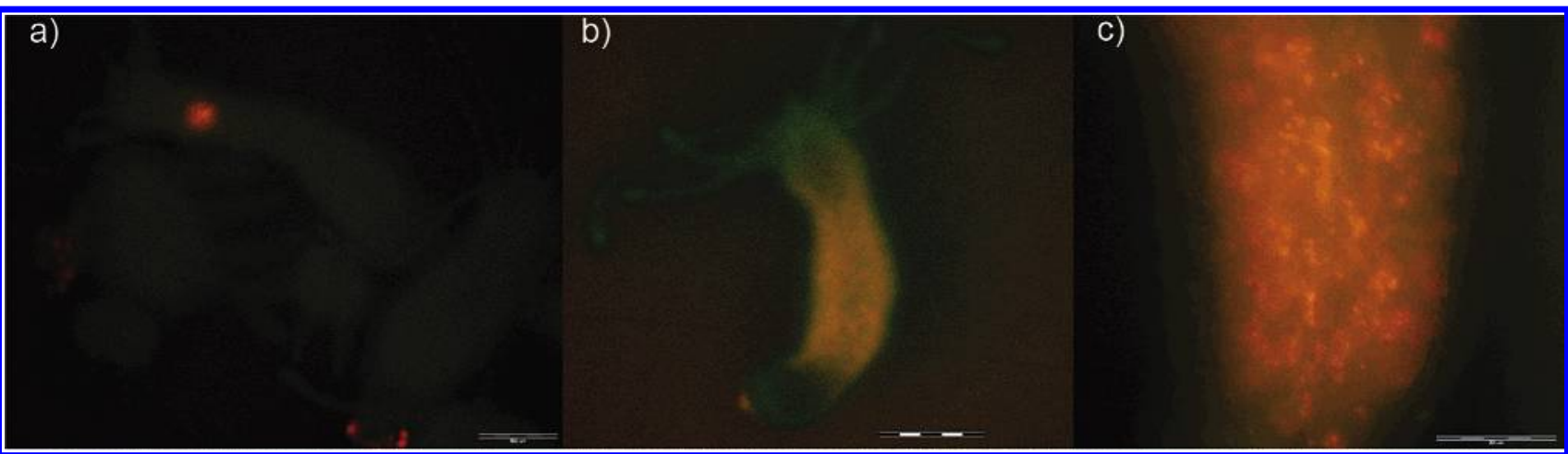

Figure 2. In vivo fluorescence imaging of Hydra polyps treated with $300 \mathrm{nM} \mathrm{GSH-QDs}$ (emission max: $610 \mathrm{~nm}$ ). (a) Image taken $1 \mathrm{~h}$ after treatment. A fluorescent stain is located inside the body and can move along it. (b) $24 \mathrm{~h}$ after treatment, an intense fluorescence is distributed all along the gastric region. (c) Enlargement of the body column showing clearly the signal inside the body of the animal. The plane of focus lies close to the outer body surface. Scale bars: $500 \mu \mathrm{m}$ (a,b) and $200 \mu \mathrm{m}$ (c). The same results where observed when the feeding activator GSH (10 $\mu \mathrm{M})$ was added to the bathing solution, stimulating mouth opening and allowing the nanoparticles to reach the gastrovascular cavity.

structure of the intact tripeptide backbone of glutathione, while tripeptide analogues with large and charged substituents at the sulfydryl group of glutathione competitively inhibit glutathione action. It is worth noting that the conversion of the oxidized form of gluthatione (GSSG) to its reduced form is crucial not only to ensure the activation of the feeding response but also because GSSG is actually known to act as an inhibitor of the feeding response (20). Therefore, before studying the interaction of GSH-QDs with Hydra, we always carried out a treatment of the GSH-QD sample with a reducing agent, in this specific case, DTT.

Activity of GSH-QDs on Living Hydra. The biological activity exerted by GSH-QDs and the putative cell targets were assayed in living Hydra by incubation of groups of animals separately with either polymer-coated QDs (PC-QDs) or PEGQDs or with GSH-QDs. In a first series of experiments, the hydra bath was exposed only to quantum dot samples (either PEG-QDs or GSH-QDs), while in a second series of experiments, $10 \mu \mathrm{M}$ GSH was also added together with the nanoparticles. At this concentration, the feeding response (i.e., tentacle writhing and mouth opening) was induced, allowing the medium that bathes the polyps to enter the body cavity. This assay, modified from Lenhoff (21) by Pierobon (22), and used as a reliable and quantifiable assay for study of aminoacid neurotransmitters in Hydra, allowed us to investigate the putative effect of the functionalized nanoparticles on the endodermal cells.

When the Hydra bath contained GSH-QDs, after incubation for $1 \mathrm{~h}$ at room temperature, fluorescence was detected in a small number of animals inside the body, as shown in Figure 2a. After $24 \mathrm{~h}$ of incubation at $18{ }^{\circ} \mathrm{C}$, this fluorescent signal was spread inside the whole animal body (Figure 2b). By zooming in on the Hydra body, as shown in Figure 2c, the fluorescent regions always appeared out of focus, indicating that the fluorescent material was located inside the body cavity. At this stage, it was not possible to determine whether internalization of the nanoparticles from endodermal cells had occurred or whether the fluorescent material had simply filled the body cavity. The percentage of animals showing this phenotype was equal to $15 \%$ when using GSH-QDs, while no animals showed a similar phenotype when using differently coated QDs (PCQDs and PEG-QDs) (see below). The percentage of Hydras able to stably internalize the nanoparticles increased up to $71 \%$ when in a parallel experiment $10 \mu \mathrm{M}$ GSH was added together with the GSH-QDs to the Hydra bathing solution (Figure 2). In both cases, in fact, the fluorescence pattern and intensity lasted unaltered until the animals were fed again, after which the signal started to face slowly and was diluted throughout the emerging buds. To investigate the possible uptake mecha- nisms of QDs from endodermal cells, the same experiment was repeated at the same conditions, but the incubation was carried out at $4{ }^{\circ} \mathrm{C}$, when endocytosis mechanisms, among all, are inhibited. After a $24 \mathrm{~h}$ incubation time, no spreading of the fluorescence inside the Hydra body was observed and the quantum dots remained localized in large aggregation spots inside the animal body (data not shown). To establish whether there was any concentration dependence in the uptake, GSHQD conjugates were tested at different concentrations, ranging from 3 to $400 \mathrm{nM}$. Higher concentrations (up to $0.8-1 \mu \mathrm{M}$ ) were shown to affect animal vitality, as they induced irreversible death. It was observed that the optimal concentration of GSHQDs required to stain the highest percentage of Hydra was 300 $\mathrm{nM}$. When $10 \mu \mathrm{M}$ GSH was also added to the bath, the concentration of GSH-QDs was kept constant at $300 \mathrm{nM}$.

Selective Uptake of GSH-QDs. In order to study more in detail the cellular localization of the nanoparticles that had entered into the body cavity, two types of experiments were performed. A first type of experiment is based on the fluorescence microscopy analysis of Hydra tissue slices obtained by standard cryosectioning of whole, stained animals. Figure 3a shows an optical image of the Hydra transverse section. In the image a thin cell spacer, called mesoglea (forming a line in the middle of the section) clearly separates two types of tissues: the endodermal cell layer (in Figure $3 \mathrm{a}$ is the region above the mesoglea, on the upper left region of the figure) and the ectodermal layer (on the right edge of the same figure). In Figure $3 \mathrm{~b}$, DAPI staining defines the position of the nuclei of the same cross sections, while in Figure 3c, the corresponding fluorescence derived from the QDs is shown. Figure 3d, obtained by merging Figure $3 \mathrm{~b}$ with Figure 3c, shows the locations of both the nuclei and the QDs and confirms that the fluorescence from the QDs is located in the endodermal cells, probably in the cytoplasm. A subcellular location of QDs was not possible with this procedure. A second proof of the cell internalization of the GSH-QDs was provided by fluorescence imaging of the stained polyps after tissue dissociation. By applying the procedure of Flick and Bode (18) (see Materials and Methods section), stained Hydra were dissociated into single cells or clusters of cells and immediately observed under a fluorescence microscope. This procedure, although it does not allow us to define the cell type, confirmed the location of the QDs fluorescence inside the cells, as shown in Figure 4.

PC-QDs and PEG-QDs are not active on Living Hydra. As control experiments, bare PC-QDs and PEG-QDs were tested on living Hydra as described above. No animals showed a fluorescence phenotype similar to that observed with GSHQDs. Instead, a more localized fluorescence was detected on the basal disk confined on the foot of the animal, as shown in 


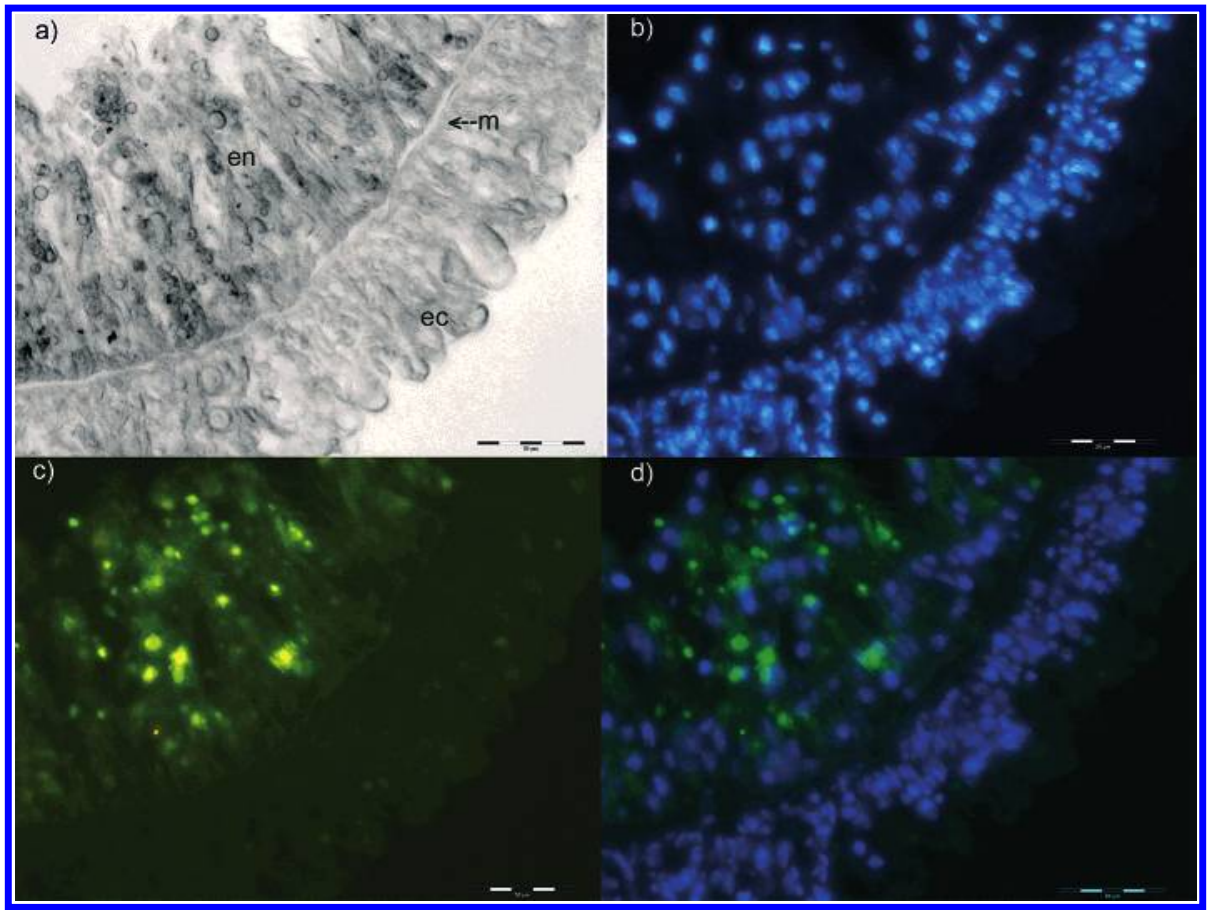

Figure 3. Cellular localization of QDs in Hydra cross sections. The whole Hydra was treated with $300 \mathrm{nM}$ GSH-QDs for $24 \mathrm{~h}$, fixed in $4 \%$ paraformaldehyde, and included for cryosectioning as described in the Materials and Methods section. Images were collected using an inverted microscope (Axiovert, 100, Zeiss) equipped with fluorescence filter sets. (a) Nomarski-DIC picture of 10 mM transversal section. Endodermal cells (en) are separated from ectodermal cells (ec) by an extracellular matrix, the mesoglea (m), indicated by the arrow. (b) DAPI staining of the same section to aim at the localization of the cell nuclei (blue color). (c) Fluorescence imaging under BP450-490/FT510/LP515 filter sets. Nanoparticles are located into endodermal cells. (d) Picture merged from parts b and c showing both DAPI (blue color) and nanoparticle (green color) fluorescence. QDs did not colocalize with nuclei.

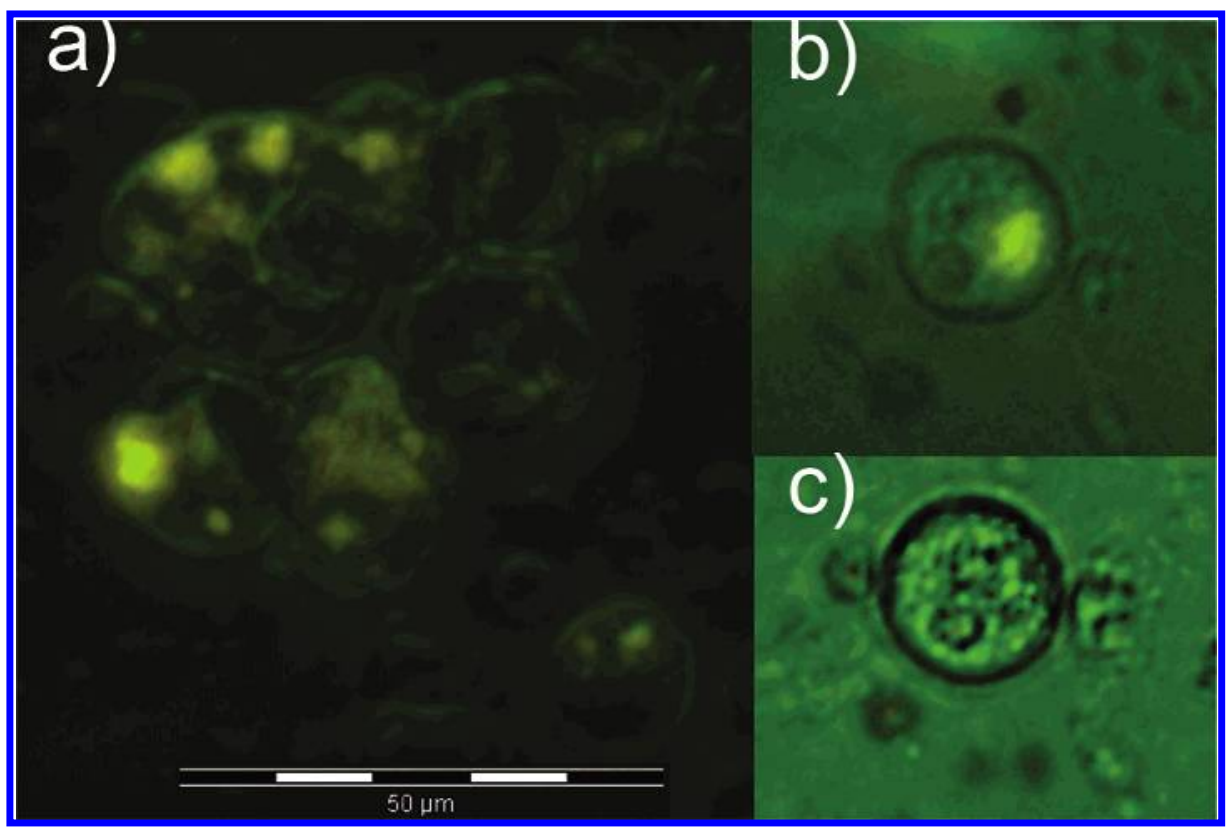

Figure 4. Dissociated cells from whole Hydra polyps treated with $300 \mathrm{nM}$ GSH-QDs for $24 \mathrm{~h}$. Single and clustered cells show nanoparticle fluorescence (yellow) inside the cytoplasm. The picture is merged from Nomarski-DIC and fluorescence images. Scale bar $=50 \mu \mathrm{m}$.

Figure 5a, 5b. These figures report bright-field and fluorescence images of a Hydra treated with PC-QDs. The same phenotype was still observed on Hydra treated with PEG-QDs, as shown in Figure 5c. QDs binding to the basal disk might depend on the sticky properties of the mucous secreted in this region, which serves to anchor the animal to a substrate. A further control experiment was carried out in parallel to evaluate the effect of PC-QDs and PEG-QDs on the endodermal cells. $10 \mu \mathrm{M}$ GSH was added to the Hydra bathing medium together with the QDs in order to induce mouth opening and to allow QDs to enter the body cavity. No internalization was observed in the case of PC-QDs, while an effect similar to that observed with GSHQDs was found with PEG-QDs. In fact, after $1 \mathrm{~h}$ of incubation, fluorescent material was detected in the body lumen, and it spread inside the animal body over the following $24 \mathrm{~h}$. However, the fluorescence lasted only $48 \mathrm{~h}$, after which it became weaker until it almost faded completely. In this set of experiments, only $27 \%$ of the Hydra showed fluorescence signal. Table 1 reports a summary of the different trials that were performed. 


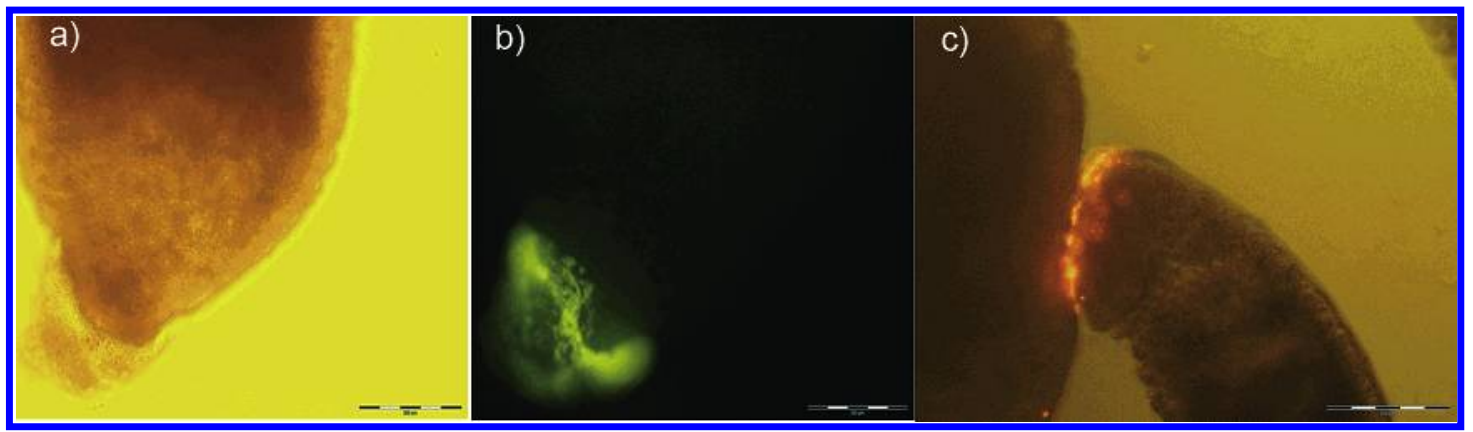

Figure 5. In vivo fluorescent imaging of Hydra polyps treated with different QDs (a) bright-field image of the Hydra peduncle and basal disc. (b) Fluorescence microscopy of the same region as in part a. Polyps were treated with PC-QDs (max emission $=510 \mathrm{~nm}$ ). Fluorescence is present in the basal disk. (c) Image merge from bright-field and fluorescence images of Hydra treated with PEG-QDs (max emission $=610 \mathrm{~nm})$. Fluorescence is present at the basal disk of a budding Hydra. Scale bar $=200 \mathrm{~mm}$.

Table 1. Number of animals, expressed as a percentage of 20 treated animals, showing endodermal fluorescent signal after $24 \mathrm{~h}$ incubation with the indicated type of QDs, with or without addition of $10 \mu \mathrm{M} \mathrm{GSH}^{a}$

\begin{tabular}{ccc} 
& free GSH added & no free GSH added \\
\hline PEG-QDs & $27 \%$ & $0 \%$ \\
GSH-QDs & $71 \%$ & $15 \%$
\end{tabular}

${ }^{a}$ The data shown are averaged over three independent experiments.

\section{DISCUSSION}

Overall, the results reported here suggest several interesting conclusions about the behavior of the Hydra when exposed to quantum dots. In the case of GSH-QDs, a fluorescent signal inside the body of the animal was observed. Because no ectodermal cells but only endodermal cells were stained by using GSH-QDs, a delivery of QDs inside the animal body must have occurred. This means that a response that culminated in mouth opening was promoted by GSH-QDs. The activity induced by GSH-QDs appeared different from the feeding behavior evoked by unbound GSH, as in fact tentacle writhing and wide mouth opening were not observed. However, such activity appears specific for GSH-QDs, as it was not induced in any of the observed cases by bare polymer-coated QDs (PCQDs) or by diamino-PEG-functionalized quantum dots (PEGQDs). Both PC-QDs and PEG-QDs were adsorbed to the mucous-producing ectodermal cells in the basal disk of living animals, but were unable to induce any behavioral response. This foot fluorescence was rapidly lost, due to the continuous cell displacement at the animal basal end. These data show that GSH-QDs alone can stimulate a response, although in a small percentage $(15 \%)$ of the treated animals. Possible reasons for this low percentage could be a low concentration of the GSH molecules conjugated to the QD surface or the modified stereochemical conformation of the bound GSH molecules, which does not allow for correct interaction with the protein target. From Lenhoff's studies (20) of the kinetics of the Hydra GSH-activated feeding response, it was shown that the duration of the feeding response increased with increasing GSH concentration, in a range from 1 to $10 \mu \mathrm{M}$. In the present work, under the experimental condition at which biological activity was observed, the concentration of GSH molecules bound to the nanoparticles was estimated to be around $5 \mu \mathrm{M}$, thus falling into the "active" GSH concentration range. Therefore, the low level of evoked activity of the GSH-QDs might be due to the fact that, once GSH is bound to the QDs, its active site either is not accessible or is not properly oriented to bind to the protein target effectively. In addition, it must be noted that, when a GSH-QD binds to the protein target via a GSH molecule, all the remaining GSH molecules located on that specific QD will no longer be available to other protein targets. Therefore, this should also account for the low level of activity observed for GSH-QDs.

Upon addition of unbound GSH to the treated animals, which allowed the nanoparticles to reach the gastric cavity, the functionalized QDs were unexpectedly taken up by the endodermal cells.

An important consideration raised by this internalization concerns the specificity of this process. Is there a specific uptake or is there a GSH-receptor-mediated process occurring in the gastric cavity? One way to address this point is by considering that, once the QDs are driven into the gastric cavity, there is a substantial difference regarding whether they carry GSH bound to their surface or not. The first difference concerns the percentage of the stained animals, which is $71 \%$ for GSHQDs and only $27 \%$ in the case of PEG-QDs. The second important difference concerns the fate of the nanoparticles taken up by the endodermal cells. Bare PEG-QDs driven into the Hydra body by free GSH are taken up by endodermal cells within $24 \mathrm{~h}$. However, $48 \mathrm{~h}$ later the fluorescence is weaker, and it becomes hard to detect a few days later. This suggests the presence of a nonspecific uptake process, probably due to endocytosis operated by endodermal cells. On the other hand, GSH-QDs, no matter how they are driven into the gastric cavity (with or without addition of free GSH), once they are taken up by the endodermal cells, show no signs of weakening over time, as they last up to a new animal feeding. These results suggest that GSH-QDs might follow a different path as compared to that followed by PEG-QDs. Furthermore, it appears likely that the GSH-mediated pathway preserves the QDs from photodegradation, as opposed to the fate of PEG-QDs, whose fluorescence is rapidly degraded.

Apart from previous studies on radioligand binding of GSH to its putative receptors in a crude membrane preparation, including membranes coming from whole Hydra vulgaris homogenates $(10,23)$, no studies of in vivo imaging of the GSH binding sites had been reported so far. Recently, Shaw et al. (24), while attempting to synthesize a fluorescent probe to study GSH receptor localization, produced a ligand that binds and antagonizes the NMDA receptor in cultured mammalian neuronal cells. To the best of our knowledge, the present study is the first one which allows localization in vivo of a GSH binding site, and we are confident that future investigations might shed light on the nature of this molecule as GSH-carrier, -receptor, or -membrane bound metabolic enzyme. Considering the multiple roles played by glutathione in metabolic functions, and in particular in the nervous system of higher vertebrates, GSHfunctionalized nanocrystals prepared and tested in this work represent promising tools for a wide variety of investigations, such as the elucidation of the role played by GSH in neurotransmission and the identification of its putative receptor. Finally, 
considering the more conventional and best-known antioxidant and free radical scavenging action of glutathione, GSH-QDs represent potentially useful redox-sensitive probes which could be used to label living cells or subcellular components according to their redox state.

\section{CONCLUSIONS}

In conclusion, we have designed and prepared GSH-functionalized quantum dot conjugates which allowed us to study the behavior of a small freshwater invertebrate called Hydra. We have tested and shown the biological activity of GSH molecules linked to fluorescent quantum dots by assaying the behavior of Hydra vulgaris.

Furthermore, by comparing the behavior of PEG-QDs in presence of free GSH to that of GSH-QDs both in the case of free GSH addition and in the absence of free GSH, we could suggest the presence of at least two mechanisms of internalization of particles of endodermal and not ectodermal cells, one relying upon particle size, charge, and surface composition, and the other, GSH-mediated, highly specific, which indicates the presence of GSH binding proteins in the endodermal cell layer. Considering the plasticity of Hydra tissues and the extensive remodeling that underlies the regeneration mechanism in this model organism, we can conclude that these photostable fluorophores represent suitable probes for this type of investigation.

\section{ACKNOWLEDGMENT}

We thank Vittorio Guglielmotti and Luigia Cristino for help with the cryostat sections and for discussion on fluorescent patterns, and Luigi Carbone, Angela Fiore, and Riccardo Di Corato for supplying QD samples. We thank also Giuseppe Ciccarella and Andrea Ragusa for helpful discussion on the characterization of samples. The authors are especially grateful to P. Pierobon for many inspiring discussions. This work was partially supported by the Italian Ministry of Research (FIRB grants RBN01KJHT-007 and RBIN048TSE, RBLA03ER38) and by the European STREP project SA-NANO (contract number 013698).

Supporting Information Available: Additional information as mentioned in the text. This material is available free of charge via the Internet at http://pubs.acs.org.

\section{LITERATURE CITED}

(1) Medintz, I. L., Uyeda, H. T., Goldman, E. R., and Mattoussi, H. (2005) Quantum dot bioconjugates for imaging, labelling and sensing. Nat. Mater. 4, 435-446.

(2) Parak, W. J., Pellegrino, T., and Plank, C. (2005) Labelling of cells with quantum dots. Nanotechnology 16, R9-R25.

(3) Michalet, X., Pinaud, F., Lacoste, T. D., Dahan, M., Bruchez, M. P., Alivisatos, A. P., and Weiss, S. (2001) Properties of fluorescent semiconductor nanocrystals and their application to biological labeling. Single Molecules 2, 261-276.

(4) Michalet, X., Pinaud, F. F., Bentolila, L. A., Tsay, J. M., Doose, S., Li, J. J., Sundaresan, G., Wu, A. M., Gambhir, S. S., and Weiss, S. (2005) Quantum dots for live cells, in vivo imaging, and diagnostics. Science 307, 538-544.

(5) Klostranec, J. M., and Chan, W. C. W. (2006) Quantum dots in biological and biomedical research: Recent progress and present challenges. Adv. Mater. 18, 1953-1964.
(6) Shaw C. A. (1998) Multiple roles of glutathione in the nervous system. Glutathione in the nervous system. (Shaw C. A., Ed.) pp 3-22, Chapter 1, Taylor \& Francis, Washington DC.

(7) Loomis, W. F., and Lenhoff, H. M. (1956) Growth and sexual differentiation of Hydra in mass culture. J. Exp. Zool. 132, 555574.

(8) Loomis, W. F. (1955) Glutathione control of the specific feeding reactions of Hydra. Ann. N. Y. Acad. Sci. 62, 208-209.

(9) Grosvenor, W., Bellis, S. L., Kass-Simon, G., and Rhoads, D. E. (1992) Chemoreception in hydra - specific binding of glutathione to a membrane-fraction. Biochim. Biophys. Acta 1117, 120-125.

(10) Bellis, S. L., Laux, D. C., and Rhoads, D. E. (1994) Affinity purification of hydra glutathione binding-proteins. FEBS Lett. 354, 320-324.

(11) Peng, Z. A., and Peng, X. G. (2001) Formation of high-quality $\mathrm{CdTe}, \mathrm{CdSe}$, and $\mathrm{CdS}$ nanocrystals using $\mathrm{CdO}$ as precursor. $\underline{\mathbf{J} . \mathrm{Am}}$. Chem. Soc. 123, 183-184.

(12) Dabbousi, B. O., Rodriguez-Viejo, J., Mikulec, F. V., Heine, J. R., Mattoussi, H., Ober, R., Jensen, K. F., and Bawendi, M. G. (1997) $(\mathrm{CdSe}) \mathrm{ZnS}$ core-shell quantum dots: Synthesis and characterization of a size series of highly luminescent nanocrystallites. J. Phvs. Chem. B 101, 9463-9475.

(13) Pellegrino, T., Manna, L., Kudera, S., Liedl, T., Koktysh, D., Rogach, A. L., Keller, S., Radler, J., Natile, G., and Parak, W. J. (2004) Hydrophobic nanocrystals coated with an amphiphilic polymer shell: A general route to water soluble nanocrystals. Nano Lett. 4, 703-707.

(14) Yu, W. W., Qu, L. H., Guo, W. Z., and Peng, X. G. (2003) Experimental determination of the extinction coefficient of CdTe, CdSe, and CdS nanocrystals. Chem. Mater. 15, 2854-2860.

(15) Williams, A., and Ibrahim, I. T. (1981) Carbodiimide chemistry: recent advances. Chem. Rev. 81, 589-636.

(16) Riddles, P. W., Blakeley, R. L., and Zerner, B. (1979) Ellman's reagent: 5,5'-dithiobis(2 nitrobenzoic acid)- a reexamination. Anal. Chem. 94, 75-81.

(17) Hermanson, G.-T. (1996) Bioconjugate Techniques. Modification of Nucleic acids and oligonucleotides. (Hermanson, G.-T., Ed.) pp 77-79, Chapter 3, Academic Press, San Diego.

(18) Flick, K. M., and Bode, H. R. (1983) Dissociating tissues into cells and the development of Hydra from aggregated cells. Hydra: research methods. (Lenhoff, H. M., Ed.) pp 251-259 Chapter 32, Plenum Press, New York.

(19) Sperling, R. A., Pellegrino, T., Li, J. K., Chang, W. H., and Parak, W. J. (2006) Electrophoretic separation of nanoparticles with a discrete number of functional groups. Adv. Funct. Mater. 16, 943948.

(20) Lenhoff, H. M., and Bovaird, J. (1961) Action of glutamic acid and glutathione analogs on the Hydra glutathione receptor. Nature (London) 189, 486-487.

(21) Lenhoff, H. M. (1961) Activation of the feeding reflex in Hydra litoralis. I. Role played by reduced glutathione and quantitative assays of the feeding reflex. J. Gen. Phvsiol. 45, 331-344.

(22) Pierobon, P., Sogliano, C., Minei, R., Tino, A., Porcu, P., Marino, G., Tortiglione, C., and Concas, A. (2004) Putative NMDA receptors in Hydra: a biochemical and functional study. Eur. J. Neurosci. 20, $2598-2604$.

(23) Bellis, S. L., Kasssimon, G., and Rhoads, D. E. (1992) Partial characterization and detergent solubilization of the putative glutathione chemoreceptor from hydra. Biochemistry 31, 9838-9843.

(24) Shaw, C. A., Pasqualotto, B. A., Curry, K., Kim, S. U., LeCompte, K. A., and Langmuir, M. E. (1999) A novel fluorescent GSH-adduct binds to the NMDA receptor. J. Neurosci. Methods 93, 21-26.

BC060355T 